\title{
If you are not prepared to be wrong, then your self-serving biases are simply not enough. Relationships between self-serving biases and discrepant self-esteem.
}

Cristina Zogmaister,

Psychology Department,

Università di Milano-Bicocca

Piazza dell'Ateneo Nuovo, 1 - 20126 Milano (Italy)

Laurentiu P. Maricutoiu,

Psychology Department,

West University of Timişoara

Bulevardul Vasile Pârvan 4, - 300223 Timișoara (Romania)

\begin{abstract}
Previous research has demonstrated that individuals with discrepant (fragile: high explicit and low implicit, or damaged: high implicit and low explicit) self-esteem are more likely to engage in defensive mechanisms than individuals with consistent implicit and explicit self-esteem. With two studies, we investigated the relationship between implicit and explicit self-esteem, and three defensive strategies for the threat of failure: subjective overachievement, psychological disengagement, and retroactive excuses. In Study $1(\mathrm{~N}=176)$, fragile self-esteem was associated with subjective overachievement, and low implicit self-esteem was associated with psychological disengagement. In Study $2(\mathrm{~N}=101)$, damaged self-esteem was related to increased use of retroactive excuses as a form of self-serving bias. These results add to the growing body of evidence documenting the maladaptive nature of fragile and damaged self-esteem.
\end{abstract}




\section{Keywords}

Implicit self-esteem; Explicit self-esteem; psychological disengagement; subjective overachievement; self-serving bias; attributional bias; retroactive excuses; self-esteem discrepancies

Short title: Relationships between self-serving biases and discrepant self-esteem

\section{Highlights}

- We investigated the relationship between defensive reactions and self-esteem

- Making excuses was related to damaged self-esteem (low explicit, high implicit)

- Overachievement was associated with fragile self-esteem (high explicit, low implicit)

- Disengagement was negatively related to implicit self-esteem

- Overachievement and disengagement emerged as independent reactions to failure. 


\section{If you are not prepared to be wrong, then your self-serving biases are simply not enough. Relationships between self-serving biases and discrepant self-esteem}

In everyday life, we encounter challenging tasks, and at times our performance falls short of our expectations or wishes. Facing failure is a challenge, both from the practical and from the psychological point of view. For instance, a student who fails an exam has to form a strategy to achieve a better level of preparation, but also to manage the possible implication of the result for the self-evaluation. Indeed failing, or performing below one's expectations, can be a threat to our sense of self-worth and is associated with adverse outcomes in terms of psychological well-being. Longitudinal studies connect failure in adolescence with clinical depression in adulthood [1], and important failures on one's job may engender negative reactions such as shame and anxiety, which in turn can enhance the risk of further mistakes [2].

When an adverse outcome suggests that we may lack abilities that we consider of value, this poses a threat to our self-esteem and to the motivation of self enhancement, that is, to maintain, boost, and preserve a positive self view [3]. Individuals enact various defensive strategies to protect themselves from the negative psychological consequences of failure $[4,5]$. For instance, they may put excessive amounts of energy and resources in the task, in order to ensure success (subjective overachievement), diminish the importance of the failure and express disinterest in the domain in order to detach self-esteem from the outcome (psychological disengagement), or make excuses for the negative outcome (self-serving attributional bias). These strategies, however, can be dangerous in the long term, because they hinder a correct appraisal of our strengths and, in the end, our ability to make appropriate life decisions. It is, therefore, important to understand what are the characteristics of persons that are linked to the use of these defensive strategies.

Indeed, previous literature reviews concluded that individuals have a universal positivity bias in how they handle self-relevant information [6], and that bias is enhanced by the existence of negative self-descriptive information [4]. Importantly, because the negative self-descriptive 
information threatens one's positive self-evaluation (i.e., self-esteem), individuals with high selfesteem showed stronger self-serving attributional biases [4]. However, more recent theoretical developments suggested that the positive relationship between self-esteem and the strength of attributional biases is not as straightforward as it was initially presumed. According to the fragile versus secure high self-esteem perspective [7-9], the self-serving biases are stronger in individuals with fragile high self-esteem, as compared with individuals with secure self-esteem.

In the present research, we contribute to the understanding of the relationship between selfesteem and three widespread, but dysfunctional strategies that one may adopt in front of challenging situations: subjective overachieving, psychological disengagement, and self-serving attributional bias.

\section{Subjective overachievement}

Subjective overachievement [5] consists in putting an enormous effort into one's strive for success. Overachievers protect themselves from the menace posed by the negative implications of failure by avoiding failure tout court. However, this comes with a cost for self knowledge: This excessive outflow of effort in the task renders performance less informative regarding one's abilities because success can be explained away by the exceptional effort. In such a way, it does not allow overachievers to gather important information about their strengths and weaknesses. This strategy is, in a sense, opposite to psychological disengagement $[10,11]$, or domain disidentification, which is a defensive mechanism used to diminish the importance of the failure [12].

\section{Psychological disengagement}

Psychological disengagement has been largely investigated in intergroup relations. It has been convincingly shown that membership in a stigmatized group that is stereotypically associated with bad performance in a given domain (i.e., stereotype threat; [13]) may lead to devaluation of the domain or discounting of the negative feedbacks [14]In this way, self-esteem remains unaffected by failure in that domain [13]However, performance and intrinsic motivation are negatively impacted. 
Opposite to overachievers, disengagers withdraw their effort $[15,16]$. If overachievers jeopardize their possibility to claim their abilities thanks to their successes, disengagers jeopardize their possibility of success in the domain they have disengaged from.

\section{Self-serving bias}

Another way to deal with external feedback about failure is by resorting to self-serving biases. The term self-serving bias refers to "external attributions for outcomes that disfavor the self but internal attributions for outcomes that favor the self" ([4], p. 23). These attributions are motivated by the individuals' need to protect or to enhance their self-esteem [6], and systematic literature reviews found different variables that explain their occurrence. The self-serving biases are stronger when the task is important for the self, when self-esteem is high, when the individual has self-focused attention and is in a positive mood [4].

It is important to note that self-serving attributional bias is an umbrella concept referring to different forms of causal attributions, which range from taking credit for positive outcomes, to making external attributions, or internal but unstable attributions for negative outcomes. Albeit, in general, a positive relationship has been evidenced between self-serving bias and self-esteem [4], a more articulated view may suggest that specific, and more problematic, self-serving biases might be characteristic of individuals with low self-esteem. Indeed, [17] found qualitative differences in the way individuals with high and low self-esteem reported negative autobiographical accounts. Individuals with high self-esteem were more likely to focus on the behavior, in an attempt to reframe it as less negative, while those characterized by low self-esteem more readily accepted the negativity of the behavior but more often resorted to external attributions, suggesting the presence of different self-presentation goals. Importantly, as noted by Schutz ([17] p. 474): “The strategies used by HSEs [high self-esteem] and LSEs [low self-esteem individuals] may be associated with different consequences for people's self-perceptions and may, in fact, perpetuate high or low selfesteem(...) Excuses, the preferred defensive strategy of LSEs, may be effective in preventing blame, but they may cost dearly." In a similar vein, [18] found a low negative correlation between 
questionnaire measures of self-esteem and of the use of retroactive excuses (e.g., "In front of a failure, you can say that the test is too difficult"), in a large sample of high-school students. This inconsistency is contrary to the general expectation that self-serving bias is more pronounced in individuals with high self-esteem and might be related to the specific type of self-serving bias. With the present research, we will focus on one specific type of self-serving attribution bias, namely the use of retroactive excuses to attribute a negative outcome to external causes.

Different from psychological disengagement, self-serving external attributions of failure can be made while maintaining interest in the domain and giving importance to the related abilities, but they are nevertheless related to costs in terms of objective appraisal and knowledge of one's strengths and weaknesses

In sum, subjective overachievement, psychological disengagement, and self-serving retroactive excuses are dysfunctional strategies that associate the protection of the self-image with costs in terms of self knowledge. They may be particularly problematic at young ages, during adolescence and early adulthood. These are critical phases of life, in which individuals are construing their self concept, and self doubt can have important life consequences [19]At such young ages, the request to be successful can be particularly exacting, as individuals face the challenge to form a coherent sense of identity and build a positive self-esteem and self-efficacy [1923]. Especially in these life phases, inadequate strategies hinder the possibility to solve one's self doubts and form a solid sense of one's abilities, and may be detrimental.

\section{Self-esteem and defensive strategies}

Empirical evidence indicates that individuals characterized by lower self-esteem suffer more emotional distress when they encounter or anticipate negative feedback [24-26]. It is, therefore, conceivable that, in order to protect themselves from the harm caused by failure and its implications, they have a higher tendency to resort to defensive strategies. The use of these strategies, in turn, makes it harder to gauge one's skills, and therefore hinders the creation of a solid 
sense of self-worth. For these reasons, in general, we should expect a negative relationship between self-esteem and the use of defensive strategies.

When considering the relationship between defensive mechanisms and self-esteem, however, we have to take into consideration the distinction between its implicit and explicit expressions. By explicit self-esteem (ESE) we mean the conscious evaluation of one's self-worth based on an intentional self-appraisal, which is typically investigated through questionnaires, as the Rosenberg Self Esteem Scale (RSES; [27]). By implicit self-esteem (ISE) we refer to the more spontaneous and automatic affective reactions toward the self [28], that are generally estimated through associative measures like the Implicit Association Test [29] or spontaneous evaluations of stimuli associated to the self, most notably the initials of one's name in the Name Letter Task (NLT; [30]). These two expressions of self-esteem can be inconsistent with each other [31, 32], and this inconsistency is thought to be maladaptive [33]. Some individuals describe themselves in highly favorable terms in their consciously controlled self-evaluations, but their automatic affective reactions suggest a less positive implicit self-esteem: Their self-esteem is considered fragile, insecure, and vulnerable to threat. This personality setup has been shown to be associated with unrealistic optimism and an idealized view of the self [28], and with an increased tendency to process threatening information with anger and hostility, which is considered a defensive reaction [34]. There is substantial agreement on the notion that people with fragile self-esteem are more easily threatened by failure and less readily accept their bad qualities, as compared to individuals with secure high self-esteem [28].

The other situation of inconsistency is when individuals hold low ESE and high ISE, or in other words, show a lower than average self regard in responses to questionnaires, but at the same time display high positivity in their automatic reactions to the self-concept. This condition has been labeled damaged self-esteem, because it is thought to be present in individuals who initially had high self-esteem, but could not meet their high self-expectations and, therefore, lowered their 
conscious self-regard. This pattern of inconsistent self-esteem, too, has been related to enhanced defensiveness [35].

It has been suggested that self-serving biases be stronger when self-evaluations are discrepant [7]. This notion has been supported by empirical evidence showing that inconsistency between ISE and ESE was related to various defense mechanisms, as giving more importance to those attributes that the individual believes to possess [36], increased outgroup derogation [36], positive reactions to ambiguous statements [35], and more superficial examination of negative feedback [35]. Based on the reasoning that both fragile/insecure self-esteem and damaged selfesteem are related to defensive responses $[28,34,35]$, it is conceivable that ISE-ESE inconsistencies are related to the three defensive strategies discussed above. No study, however, has systematically investigated the relationship between subjective overachievement, psychological disengagement, and self-serving attributions, on the one hand, and ISE, ESE, and their interaction on the other hand. With this research, we aim to investigate these aspects.

\section{The research}

We describe two studies, in which we investigated these strategies in adolescence and emerging adulthood. In Study 1, in a sample of adolescents, we investigated how ESE, ISE, and their interplay, related to general tendencies to overachievement and psychological disengagement, focusing on evidence regarding the general tendency to use these defensive strategies. Next, in Study 2 we investigated the occurrence of this general tendency in a real failure situation. Therefore, we investigated a sample of early adults who received negative feedback regarding their ability to solve a logical task, and put their measured ESE, ISE, and their interplay, in relation to self-serving attributions to this feedback.

\section{Study 1}

\section{Method}

\section{Participants}


We contacted a total of 204 adolescents in two Italian high schools. In the case of minors, also their parents were given a brief description of the research and asked to consent to participate. In four cases, we did not receive the consent of the parents or of the students, and two students could not complete the study for time reasons. The final sample consists of 198 Italian adolescents ( $75.77 \%$ female, mean age 17.55 years), who volunteered to take part in this study. The size of the sample is based on convenience reasons. A sensitivity analysis, conducted with G*Power [37] with significance level $\alpha=.05$, power $(1-\beta)=.80$, based on the F-test family for multiple regression with three predictors indicated that we had an adequate level of power for an effect size of $f^{2}=.054$, which is considered small-to-medium [38].

\section{Measures}

Implicit Self Esteem. We presented participants the NLT, in which they rated the letters from A to $Z$, in a fixed random order, on 10-point scales from $1=I$ don't like it at all to $10=I$ like it very much. We chose a 10-point scale to make it more similar to the Italian school evaluation system, which uses marks from 1 to 10 , where 10 is the best result. We computed ISE scores with the Ialgorithm [30] that corrects both for differences in normative pleasantness of the letters and individual differences in baseline response tendencies in letter ratings. Higher scores indicated higher ISE

Explicit Self Esteem. We used the Italian translation of the RSES [39]. Participants evaluated each of the RSES sentences on a 6-point scale from 1 (strongly disagree) to 6 (strongly agree)The RSES is a widely used self-report measure of global trait Self Esteem and contains an equal number of positively (e.g., On the whole, I am satisfied with myself) and negatively worded items (e.g., At times I think I am no good at all)We computed ESE as the summed score of the responses, after reverse scoring the negatively worded items. Higher scores indicated higher levels of ESE

Self doubt and Concern with Performance. We presented participants with the Subjective Overachievement Scale (SOS; [5]; translated in Italian for the present research), a 17-item questionnaire which includes two independent subscales: concern with performance and self doubt. 
The Concern with Performance subscale consists of nine statements regarding the concepts of success and personal failure (e.g., It is important that I succeed in all that I do; I try to avoid being too successful). The self doubt subscale consists of eight statements about the perception of one's own value and competence. These refer to the general sense of being unsure about one's own capabilities and the desire to avoid negative outcomes, without any specific reference to coping strategies (e.g., More often than not I feel unsure of my abilities). Self doubt does not imply the belief of being incapable of performing certain tasks: It is better described as insecurity regarding one's level of competence. As stated by Braslow and colleagues ([40], p. 472): “chronically self doubtful individuals might be viewed as having a wide confidence interval around judgments of their ability." It is normal to experience self doubt sometimes, but chronic self doubt is problematic, as it is negatively related to well-being and it is considered as enhancing the sensitivity to the implications of outcomes for the self, which can have a generally negative impact on performance, by diverting attention from the task and focusing it on the self [40]. Self doubt it thought to trigger self-defensive strategies to cope with difficult tasks, most notably self-handicapping and overachieving. Empirical results have evidenced a relatively high negative correlation between self doubt and ESE $[5,41]$. Given that previous research had shown its relation with self-serving strategies, we deemed it worth also exploring its relation with ISE and with the ISE-ESE interplay. Participants evaluated each of the SOS sentences on a 6-point scale from $1=$ strongly disagree to 6

= strongly agree

Procedure

All participants completed a paper and pencil questionnaire composed of the measures described above, in the following order: the NLT, the RSES, and the SOS. Next, we presented participants with a questionnaire on alcohol consumption, unrelated to the scopes of the present research, and in the end we asked respondents to report their age, gender, and the first two initial letters of the given name. In order for the students to feel their privacy protected, we did not ask the initial of their surname 


\section{Results}

\section{Preliminary analyses}

Twenty-one participants failed to evaluate one or more letters in the NLT and we eliminated them from the analysis (15 participants failed to evaluate one letter, two participants failed to evaluate two letters, one participant three letters, and three participants evaluated none of the letters)

We computed the internal consistency of the self-report scales (see Table 1). The RSES showed adequate internal consistency. The Self Doubt subscale of SOS showed acceptable internal consistency and the Principal Component Analysis (PCA) indicated that this subscale had a single component structure. For the Concern with Performance subscale, the PCA indicated the presence of two orthogonal components (oblimin rotation indicated a correlation of $r=.215$ between the two components). We interpreted them as Psychological Disengagement ("There are some situations where I think it is better that I fail", "I think that in some situations it is important that I not succeed", "Sometimes I am more comfortable when I lose or do poorly", and "I try to avoid being too successful") and Psychological Overachievement ("Failure is unacceptable to me", "It is important that I succeed in all that I do", "I strive to be successful at all times", "For me, being successful is not necessarily the best thing" (R)). We considered the internal consistency of these two components acceptable, considering that they were based on a restricted number of items, and computed the summed scores for these two components.

We used the 2-MAD rule to investigate potential outliers. Based on the subsequent visual inspection of the histogram of frequencies, we detected five outliers for the variable ISE, and two outliers for the variable ESE, each distant from the median more than five MADs. These data were eliminated from the analysis listwise, leaving a final sample of 176 participants. Table 1 reports the main descriptive statistics and correlations between the variables.

Table 1. Main descriptive statistics and correlations for the variables of Study 1. 


\begin{tabular}{|c|c|c|c|c|c|c|c|c|c|}
\hline & \multicolumn{4}{|c|}{$\underline{\text { Descriptive statistics }}$} & \multicolumn{5}{|c|}{$\underline{\text { Correlation matrix }}$} \\
\hline & Min & Max & Mean & $\mathrm{SD}$ & 1. & 2. & 3. & 4. & 5. \\
\hline $\begin{array}{l}\text { 1Implicit Self Esteem } \\
\text { (NLT) }\end{array}$ & -2.667 & 6.832 & 2.816 & 1.870 & - & & & & \\
\hline $\begin{array}{l}\text { 2Explicit Self Esteem } \\
\text { (RSES) }\end{array}$ & 21 & 59 & 44.213 & 7.766 & .078 & .832 & & & \\
\hline 3Self Doubt & 12 & 44 & 29.432 & 6.345 & .008 & $-.608 * * *$ & .734 & & \\
\hline 4Overachievement & 6 & 24 & 17.674 & 3.921 & .075 & $.217^{* *}$ & $-.329 * * *$ & .689 & \\
\hline 5Disengagement & 7 & 24 & 15.414 & 3.965 & $-.155^{*}$ & .025 & -.070 & $.247 * *$ & .640 \\
\hline
\end{tabular}

Note $* \mathrm{p}<.05, * * \mathrm{p}<.01, * * * \mathrm{p}<.001 . \mathrm{N}=176$. Internal consistency indices for each measure are presented on the diagonal.

Relationship between self-esteem and defensive strategies.

We investigated the relationship between ISE, ESE, their interplay, and the three criteria of self doubt, overachievement, and disengagement. We performed three separate hierarchical regression analyses, entering the ISE and ESE scores in the first step, and their interaction in the second step. All variables were standardized before the analysis. As indicated in Table 2, self doubt was significantly associated with ESE, but no significant relationship emerged with ISE and with the ESE*ISE interaction. The higher the ESE score, the lower the level of self doubt. Disengagement, on the other hand, was significantly associated with ISE, and an increase in ISE was accompanied by lower levels of disengagement; no relationship emerged between disengagement and either ESE, or the ESE * ISE interaction. For overachievement, ESE revealed a significant effect in Step 1, indicating that higher levels of ESE were accompanied by higher overachievement scores, whereas ISE was not significantly associated with this criterion in Step 1In Step 2, a significant ESE*ISE interaction effect emerged. A simple slope analysis indicated that 
ESE was significantly related to overachievement for participants with low levels of ISE (1 SD below the mean), $\beta=0.394, \mathrm{SE}=0.104, \mathrm{p}<.001$, and for participants with intermediate levels of ISE, $\beta=0.180, \mathrm{SE}=0.076, \mathrm{p}=.019$, and in both cases higher levels of ESE were related with higher levels of overachievement. On the other hand, for participants with higher ISE score (1 SD above the mean) no significant relation between ESE and overachievement emerged, $\beta=-0.034$, SE $=0.124, \mathrm{p}=.782 \mathrm{This}$ indicated that high ISE worked as a buffer, protecting participants from overachievement tendencies.

Table 2. Hierarchical regression on the three criteria.

\begin{tabular}{|c|c|c|c|c|c|c|}
\hline & \multicolumn{2}{|l|}{ self doubt } & \multicolumn{2}{|c|}{ Overachievement } & \multicolumn{2}{|c|}{ Disengagement } \\
\hline & $\beta(\mathrm{SE})$ & $\mathrm{R}^{2}$ & $\beta(\mathrm{SE})$ & $\mathrm{R}^{2}$ & $\beta(\mathrm{SE})$ & $\mathrm{R}^{2}$ \\
\hline Step 1 & & .368 & & .050 & & .025 \\
\hline ESE & $-.608 * *(.062)$ & & $.213 * *(.076)$ & & $.037(.077)$ & \\
\hline ISE & $.056(.0062)$ & & $.058(.076)$ & & $-.158 *(.077)$ & \\
\hline Step 2 & & .369 & & .085 & & .026 \\
\hline ESE & $-.602 * *(.063)$ & & $.180 *(.076)$ & & .033 & \\
\hline ISE & $.052(.062)$ & & $.078(.075)$ & & $-.155 *(.078)$ & \\
\hline ESE*ISE & $.040(.071)$ & & $-.214 *(.086)$ & & $-.028(.089)$ & \\
\hline$\Delta \mathrm{R}^{2}$ & & .001 & & .034 & & .001 \\
\hline F-change & $\mathrm{F}(1,165)=.308, \mathrm{p}$ & $=.580$ & $F(1,165)=6.214$ & $\mathrm{p}=.014$ & $F(1,165)=.102$ & $0=.750$ \\
\hline
\end{tabular}




\section{Discussion}

The present results indicate that avoiding success and excessively striving for success are not opposite reactions but, instead, separate defensive mechanisms. This result is consistent with empirical evidence that cognitions underlying the intention to perform a behavior and those underlying the intention not to perform it are not opposite, because they rely on separate decisionmaking processes [42]. The two dimensions of subjective overachievement and psychological disengagement that emerged from the PCA, showed different patterns of relationship with selfesteem: Psychological disengagement decreased with the increase of ISE and was not related to ESE. Subjective overachievement, on the contrary, was positively correlated with ESE; however, the relationship between overachievement and ESE was strongest for individuals characterized by low ISE, and absent for those with high ISE. These results show that the self defensive mechanism of overachievement was particularly present in those individuals characterized by a pattern of defensive, or fragile, self-esteem, or in other words by high ESE and low ISE.

Consistent with previous research [5], this study evidenced that self doubt was related to explicit self-esteem. This result is not surprising if we consider the conceptual similarity between self doubt and low levels of self clarity [43]Previous research studies confirmed that low selfesteem is strongly associated with poor self-clarity [44, 45]However, no relationship emerged between self doubt and ISE.

Having shown that ESE, ISE, and their interplay, are related to two general defensive reactions to failure, our next step consisted in focussing on the specific reactions to one episode of failure. Therefore, in Study 2 we investigated the self-serving retroactive excuses that a failure episode engendered.

\section{Study 2}

\section{Method}

Participants 
The study was conducted in a Romanian University. Participants were recruited using their institutional e-mail lists and received course credits for their voluntary participation. Out of the 124 participants enrolled in the study, 101 students (55.88\% female, mean age 22.86 years) did not find the solution to the problem-solving task and were included in the analyses. A sensitivity analysis based on $\mathrm{N}=101$, conducted with $\mathrm{G}^{*}$ Power, with significance level $\alpha=.05$, power $(1-\beta)=.80$, based on the F-test family for multiple regression with three predictors indicated that we had an adequate level of power for an effect size of $f^{2}=.11$, which is considered small-to-medium (Cohen, 1992).

\section{Measures}

Explicit and Implicit Self-Esteem. Participants were administered paper-and-pencil versions of the RSES and the NLT. Regarding the RSES, we used an existing translation that showed good reliability in previous studies on Romanian samples [46, 47]. Regarding the NLT, we presented the participants with a list of the letters sorted alphabetically, and they had to rate each letter using a 7point Likert scale ( 1 - I do not like it at all; 7 - I like it very much). Similar to Study 1 , we used the I-algorithm [30] to compute the scores of the NLT.

Attributional Biases. The criterion variables were assessed using a short self-report scale for assessing attributional biases, developed for this study. Using a 5-point Likert scale (1 - strongly disagree to 5 - strongly agree), participants rated items that assessed their tendency to attribute their result to situational factors (sample item: I did not rest well last night, which has influenced my performance in the previous task), to environmental disturbances (sample item: The noise around me constantly distracted my attention so that I could not pay attention to the task I had to fulfill), to their general inability to perform such tasks (sample item I think you need special skills to solve problems like the previous one), or simply allowed participants to minimize the importance of their failure (sample item: A single task is not enough to assess my potential). The whole list of items is presented in Table 3Because this task was specifically developed for this study, we will briefly discuss its psychometric properties in the first part of the Results section. 


\section{Procedure}

Following the completion of an informed consent form, all participants responded to paperand-pencil versions of the self-esteem measures (i.e., the RSES and the NLT). Then, we invited each participant to an individual office space, where we presented the problem-solving task. The instructions for the problem-solving task were: You have six matchsticks. Your task is to use them to form a figure that will contain 8 equilateral triangles. You are not allowed to break the matchsticks or to use other materials. You have 4 minutes to complete the task. Each participant completed the task alone, and the experimenter returned after the 4 minutes. Most participants (101 out of 124) did not solve the task, and the experimenter presented them with the solution (i.e., to form two overlapping triangles that will form the structure of the star of David). Then, participants completed the Attribution Biases scale.

\section{Data analysis}

Because we developed our outcome measures for this study, we analyzed their internal validity using a principal component analysis of the responses provided by the participants. We used parallel and screeplot analysis to determine the optimal number of factors.

For the moderation analyses, we used the Jamovi's medmod package [48] for testing mediation and moderation effects. To ease the interpretation, we standardized all variables (i.e., both self-esteem forms and the self-serving bias index), and we conducted simple slopes analyses to interpret the interaction term.

\section{Results}

\section{Preliminary analyses}

In our preliminary analyses, we investigated the internal validity of our criterion variables. We conducted a PCA. Based on parallel analysis and screeplot analysis, we extracted a single factor. Although the internal consistency of the scale containing all 16 items was acceptable (Cronbach's $\alpha=.805$ ), we excluded four items with loadings below .30 and re-analyzed the data. The remaining 12 items also grouped in a single-factor solution, and the internal consistency of this 
factor was good (Cronbach's $\alpha=.858$ ) The loadings of all items are presented in Table $3 \mathrm{We}$ computed Attributional Bias as the summed score of the responses to items in the final solution and higher values indicate a higher tendency to external attributions of the failure.

Table 3. The Attributional Biases scale: Factor loading of the initial and final solutions.

\begin{tabular}{|c|c|c|}
\hline \multirow{2}{*}{ Item } & Initial & Final \\
\hline & analysis & solution \\
\hline
\end{tabular}

11The noise around me constantly distracted my attention so that I could not pay attention to the task I had to fulfill.

$.797 \quad .796$

7The lighting mode of the room diminished my ability to solve the task.

13It was difficult for me to focus on the previous problem because of the feeling of hunger/thirst I feel.

15Today's weather has created a negative mood that has influenced the result obtained in the previous task.

9My general condition is affected by a cold (or another transient disease)

that has prevented me from giving a maximum performance to the thinking task.

3The temperature in the room made it difficult for me to concentrate on my previous task.

14The current events in my personal life captured the attention I needed to solve the thinking tasks.

2I generally have trouble keeping my focus on a task.

5If I had solved the previous task at another time of the day, my

performances would have been different. 
previous task.

4I don't think it helps with something if I know how to solve problems similar to the previous one.

10I think you need special skills to solve problems like the previous one.

12I am often put in situations where the product of my actions does not reflect my real capabilities.

$8 \mathrm{~A}$ single task is not enough to realize my potential.

6I do not face too often the need to solve problems like the previous one.

$16 \mathrm{My}$ abilities go rather to the humanities, rather than to solve the logicalmathematical problems.

\section{Moderation analyses}

Descriptive statistics and correlations between study variables are presented in Table 4Regarding the moderation analyses, we followed the analytical approach used in Study 1Results presented in Table 5 suggested that the post-task attributional bias is associated with low ESE, r(99) $=-.35, \mathrm{p}<.01$, while the regression analysis yielded a significant effect of the interaction between the two forms of self-esteem $(\beta=-.26, \mathrm{SE}=.12, \mathrm{p}=.03)$ In order to explain the interaction effect, we conducted a simple slopes analysis. We estimated the relationship between ESE and the attributional bias, for high and low levels of ISE (1 SD above and 1 SD below the mean). The simple slope analysis indicated that the relationship between ESE and attributional bias was statistically significant only when ISE was high $(\beta=-.58, \mathrm{SE}=.14, \mathrm{p}<.01)$ or medium $(\beta=-.32$, $\mathrm{SE}=.09, \mathrm{p}<.01)$, not when ISE was low $(\beta=-.06, \mathrm{SE}=.17, \mathrm{p}=.73)$.

Table 4. Descriptive statistics and correlations between study variables 


\begin{tabular}{|c|c|c|c|c|c|c|c|}
\hline & mean & SD & $\min$ & $\max$ & $\begin{array}{c}\text { Post-task } \\
\text { attributional } \\
\text { bias }\end{array}$ & $\begin{array}{c}\text { Explicit } \\
\text { self-esteem }\end{array}$ & $\begin{array}{c}\text { Implicit self- } \\
\text { esteem }\end{array}$ \\
\hline $\begin{array}{l}\text { Post-task } \\
\text { attribution } \\
\text { al bias }\end{array}$ & 2.59 & 0.77 & 1.17 & 4.42 & .87 & & \\
\hline $\begin{array}{l}\text { Explicit } \\
\text { self- } \\
\text { esteem }\end{array}$ & 3.22 & .40 & 1.90 & 4.00 & $-.35 * *$ & .82 & \\
\hline $\begin{array}{l}\text { Implicit } \\
\text { self- } \\
\text { esteem }\end{array}$ & .99 & .72 & -2.13 & 3.03 & -.02 & -.05 & - \\
\hline
\end{tabular}

$\overline{\text { Note }^{*} \mathrm{p}<.05, * * \mathrm{p}<.01 \mathrm{~N}=101 \text { Internal consistency indices for each measure are presented on the }}$ diagonal.

Table 5. Results of the hierarchical stepwise regression analyses

$\underline{\text { Post-task attributional bias }}$

$\beta(\mathrm{SE}) \quad \mathrm{R}^{2}$

Step 1

.126

$\begin{array}{ll}\text { Explicit self-esteem } & -.35^{* *}(.09) \\ \text { Implicit self-esteem } & -.03(.09)\end{array}$

Step 2

.166 


$\begin{array}{cc}\text { Explicit self-esteem } & -.32 * *(.09) \\ \text { Implicit self-esteem } & -.03(.09) \\ \text { Implicit-explicit interaction } & -.26 *(.12) \\ \text { term } & \end{array}$

$\Delta \mathrm{R}^{2}$

$$
\text { F-change } \quad \mathrm{F}(1,97)=4.62, \mathrm{p}=.03
$$

\section{Discussion}

Study 2 moved the focus from general defensive reactions to failure, to specific selfdefensive attributional biases after a situational failure. We found evidence that individuals with lower ESE showed greater use of retroactive excuses as a form of self-serving bias. Importantly, this relation between ESE and retroactive excuses differed depending on the level of ISE. For participants with medium and high ISE, lower ESE scores were associated with a higher appeal to self-serving excuses, but participants with low ISE scores showed no significant relation between ESE and this self-serving bias.

\section{General Discussion}

This research examined three defensive reactions to the threat caused by failure, namely subjective overachievement, psychological disengagement, and retroactive excuses as a self-serving bias in causal attributions of failure. The relation between ESE and defensive reactions is well documented in the literature, and empirical evidence suggested that ISE is also related to them. Previous research used both trait-like measures of defensive reactions (i.e., self-reported questionnaires of one's general tendency to respond in a certain manner) and assessments of participants' reactions to real failure situations. We, therefore, used both approaches in our research 
and showed the importance of considering both ESE and ISE, and their interplay, to better understand both general defensive reactions and attributional biases in a specific, real situation.

The correlational analyses showed that ESE was positively associated with subjective overachievement beliefs (Study 1), and negatively with the use of self-serving biases (Study 2)Importantly, in both cases the regression analysis evidenced that the relation between ESE and self defense strategy was moderated by ISE. As regards overachievement, the relation with ESE was significant only for participants characterized by low or medium ISE scores. It was nonsignificant and negligible for participants with high ISE scores. This result suggests that a high ISE plays a protective role against this defensive strategy - which in the long term can be detrimental. This finding is consistent with the general idea that individuals with secure high self-esteem do not need defenses in front of the risk of negative self-relevant information [8], while people with fragile self-esteem, because of the insecurities caused by their lower ISE, are thought to question their positive attitude toward themselves, and need frequent validation [32]. The importance of the interplay between ISE and ESE is further evidenced in Study 2, in which the increased use of retroactive excuses in participants with low ESE was present when their ISE was medium or high, but not when it was low and, therefore, consistent with ESE. This pattern of results indicates that this defensive mechanism was accentuated in case of damaged self-esteem, in line with the suggestion that individuals with damaged self-esteem may hold what has been called "a glimmer of hope" [49]: Even if their reflective evaluations of themselves are less positive than average, their more positive automatic affective reactions may stimulate them to strive to get positive results and restore their damaged Self-Esteem. As regards psychological disengagement, the present research indicated no significant relation to ESE, but this defense mechanism was negatively related to ISE, and also this result supports the importance to take into consideration this dimension of self-esteem when analyzing defense mechanisms.

Another aspect worth noting of the present results concerns the relationship between subjective overachievement and psychological disengagement. These have opposite consequences 
on behavior. The first causes an increase in the effort in order to avoid failure at any cost. The second, on the contrary, causes disinterest in success and withdrawal of effort. Using a validated scale that investigates reactions to success and failure, we showed that these two dimensions are not negatively correlated, as one would expect if they express opposite reactions. On the contrary, they were orthogonal to each other, and their independence is confirmed by the fact that they presented different patterns of relations with self-esteem. The independence of the two defensive mechanisms implies that certain individuals react to the threat of failure both with an overachieving strategy, and withdrawing their effort and interests. Further research is needed to understand if, for individuals that describe a high tendency to both overachievement and disengagement, the use of these strategies is domain-specific, or in other words, they devote all their efforts to certain domains and withdraw them from others, and how much it is subject - for a given domain - to the specific contingencies of success or failure. Anecdotal evidence from everyday experience of many educators and parents tells that some students increase their commitment after they have received a good mark, while they react by showing disinterest in the subject after a poor result, which could suggest that they make use of either strategy, to protect or enhance their self-esteem, depending on the immediate feedback.

\section{Limits and future directions}

It would be incorrect to infer that self-esteem influences the strategies we have examined, and not the other way around. We conducted correlational research; therefore, we cannot infer causality. Plausibly, however, the causal effects go both ways: On the one hand, certain configurations of self-esteem call defensive strategies, like the ones we have investigated here, as in the case in which damaged self-esteem needs to be restored or a fragile one needs to be protected. On the other hand, the use of these strategies places a veil between oneself and an honest reflection on one's abilities and characteristics, which contributes to maintaining the misalignment between the two dimensions of self-evaluation. Further longitudinal research, following the individuals over 
time, would be important to understand better the development of the relationship between selfesteem and defensive mechanisms.

We conducted this study on adolescents and early adults. In adolescence and early adulthood, individuals are forming their identity and self-esteem, therefore their self-esteem may be more fragile and their doubts about their competences higher than later on in adulthood. However, we believe that the present results are also relevant in different stages of life, and future research should investigate these effects also with older adults.

\section{Acknowledgements}

We wish to thank Silvia Testoni and Raffaella Bevilacqua for their precious help in the collection of data for Study 1.

This research did not receive any specific grant from funding agencies in the public, commercial, or not-for-profit sectors.

\section{References}

1. McCarty, CA, Mason, WA, Kosterman, R, Hawkins, JD, Lengua, LJ, McCauley, E. Adolescent school failure predicts later depression among girls. J Adolesc Health. 2008; 43: 180-187.

2. Sirriyeh, R, Lawton, R, Gardner, P, Armitage, G. Coping with medical error: a systematic review of papers to assess the effects of involvement in medical errors on healthcare professionals' psychological well-being. Qual Saf Health Care. 2010; 19: 1-8.

3. Sedikides, C, Gregg, AP. Self-enhancement: Food for thought. Perspect Psychol Sci. 2008; 3(2): 102-116. Available from: https://doi.org/10.1111/j.1745-6916.2008.00068.x

4. Campbell, WK, Sedikides, C. Self-threat magnifies the self-serving bias: A meta-analytic integration. Rev Gen Psychol. 1999;3(1):23-43. Available from: https://doi.org/10.1037/10892680.3.1.23 
5. Oleson, KC, Poehlmann, KM, Yost, JH, Lynch, ME, Arkin, RM. Subjective overachievement: Individual differences in self-doubt and concern with performance. J Pers. 2000;68(3):491-524.

6. Mezulis, AH, Abramson, LY, Hyde, JS, Hankin, BL. Is there a universal positivity bias in attributions? A meta-analytic review of individual, developmental, and cultural differences in the self-serving attributional bias. Psychol Bull. 2004;130(5):711-747. Available from: https://doi.org/10.1037/0033-2909.130.5.711

7. Kernis, MH. Substitute needs and the distinction between fragile and secure high self-esteem. Psychol Inq. 2000;11(4):298-300 Available from: https://www.jstor.org/stable/1449625

8. Kernis, MH. Toward a conceptualization of optimal self-esteem. Psychol Inq. 2003;14(1):1-26.

9. Kernis, MH, Lakey, CE. Fragile versus secure high self-esteem: Implications for defensiveness and insecurity. In: Arkin RM, Oleson KC, Carroll PJ, editors, Handbook of the uncertain self. Psychology Press; 2010. p. 360-378.

10. Laplante, J, Tougas, F, Kocum, L. A Meta-analysis of the Relationship between Psychological Disengagement and Self-esteem: The Role of Domain and Group Status. Int J Divers Organ Communities Nations: Annual Review. 2014;14:1-15.

11. Schlenker, BR, Pontari, BA, Christopher, AN. Excuses and character: Personal and social implications of excuses. Pers Soc Psychol Rev. 2001;5(1):15-32.

12. Schlenker, BR, Britt, TW, Pennington, J, Murphy, R, Doherty, K. The triangle model of responsibility. Psychol Rev. 1994;101(4):632-652. Available from: https://DOI:10.1037/0033295X.101.4.632

13. Schmader, T, Major, B, Gramzow, RH. Coping with ethnic stereotypes in the academic domain: Perceived injustice and psychological disengagement. J Soc Issues. 2001;57(1):93-111. Available from: https://doi.org/10.1111/0022-4537.00203

14. Tougas, F, Lagace, M, Laplante, J, Bellehumeur, C. Shielding self-esteem through the adoption of psychological disengagement mechanisms: The good and the bad news. The Intern J Aging Human Development. 2008;67(2):129-148. 
15. Osborne, JW. Race and academic disidentification. J Educ Psychol. 1997;89(4): 728-735. Available from: https://doi.org/10.1037/0022-0663.89.4.728

16. Osborne, JW, Rausch, JL. Identification with Academics and Academic Outcomes in Secondary Students. Paper presented at the annual meeting of the American Education Research AssociationSeattle, WA 2001. Available from: https://www.researchgate.net/profile/Jason_Osborne2/publication/234565060_Identification_wi th_Academics_and_Academic_Outcomes_in_Secondary_Students/links/57cd83fc08ae83b3746 0d76b/Identification-with-Academics-and-Academic-Outcomes-in-Secondary-Students.pdf February 21, 2020.

17. Schutz, A. Autobiographical narratives of good and bad deeds: Defensive and favorable selfdescription moderated by trait self-esteem. J Soc Clin Psychol. 1998;17(4):466-475. Available from: https://doi-org.proxy.unimib.it/10.1521/jscp.1998.17.4.466

18. Maltese, A, Alesi, M, Alù, AGM. Self-esteem, defensive strategies and social intelligence in the adolescence. Procedia Soc Behav Sci. 2012;69:2054-2060. Available from: https://doi.org/10.1016/j.sbspro.2012.12.164

19. Porfeli, EJ, Lee, B. Career development during childhood and adolescence. New Dir Youth Dev. 2012;134:11-22.

20. Arnett, JJ. Emerging adulthood: The winding road from the late teens through the twenties. 2nd ed. New York, NY: Oxford University Press; 2015

21. Erol, RY, Orth, U. Self-esteem development from age 14 to 30 years: A longitudinal study. J Pers Soc Psychol. 2011;101(3):607-619. Available from: https://doi.org/10.1037/a0024299

22. Schunk, DH, Meece, JL. Self-efficacy development in adolescence. In: Pajares F, Urdan T, editors. Self-efficacy beliefs of adolescents. Greenwich, Connecticut: Information Age Publishing; 2006, 5, 71-96. ISBN 1-59311-367-6 
23. Schwartz, SJ, Forthun, LF, Ravert, RD, Zamboanga, BL, Rodriguez, L, Umaña-Taylor, AJ....Hudson, M. The protective role of identity consolidation against health risk behaviors in college-attending emerging adults. Am J Health Behav. 2010;34:214-224.

24. Buckingham, T. Reducing contingent self-worth: A defensive response to self-threats. J Soc Psychol. 2019;159(3):284-298.

25. Krause, S, Back, MD, Egloff, B, Schmukle, SC. Predicting Self-Confident Behaviour with Implicit and Explicit Self-Esteem Measures. Eur J Pers. 2016;30(6):648-662.

26. Park, YJ, Park, JY, Chung, KM, Song, YM, Jhung, K. Discrepancies of Implicit and Explicit self-esteem as Predictors of Attributional Bias and Paranoia. Psychiatry Investig. 2019;16(3):185-192.

27. Rosenberg, M. Society and the adolescent self-image. Princeton, NJ: Princeton University Press; 1965

28. Bosson, J.K, Brown, R.P, Zeigler-Hill, V, Swann, W.B. Self-Enhancement Tendencies Among People With High Explicit self-esteem: The Moderating Role of Implicit self-esteem. Self Identity. 2003;2(3):169-187. Available from: https://doi.org/10.1080/15298860309029

29. Greenwald, A.G, Farnham, S.D. Using the implicit association test to measure self-esteem and self-concept. J Pers Soc Psychol. 2000;79(6):1022-1038 Available from: https://doi.org/10.1037//0022-3514.79.6.I022

30. LeBel, EP, Gawronski, B. How to find what's in a name: Scrutinizing the optimality of five scoring algorithms for the name-letter task. Eur J Pers. 2009;23(2):85-106.

31. Zeigler-Hill, V, Clark, CB, Beckman, TE. Fragile self-esteem and the interpersonal circumplex: Are feelings of self-worth associated with interpersonal style?. Self Identity. 2011;10(4):509536.

32. Zeigler-Hill, Vand Jordan, CH. Two faces of self-esteem: Implicit and explicit forms of selfesteem. In: Gawronski B, Payne BK, editors. Handbook of implicit social cognition: Measurement, theory, and applications, New York: Guilford Press; 2010. P. 392-407. 
33. Schröder-Abé, M, Rudolph, A, Schütz, A. High implicit self-esteem is not necessarily advantageous: Discrepancies between explicit and implicit self-esteem and their relationship with anger expression and psychological health. Eur J Pers. 2007;21(3):319-339.

34. Kernis, MH, Lakey, CE, and Heppner, WL. Secure versus fragile high self-esteem as a predictor of verbal defensiveness: Converging findings across three different markers. J Pers. 2008;76:477-512.

35. Schröder-Abé, M, Rudolph, A, Wiesner, A, Schütz, A. Self-esteem discrepancies and defensive reactions to social feedback. Int J Psychol. 2007;42(3):174-183.

36. Kernis, MH, Abend, TA, Goldman, BM, Shrira, I, Paradise, AN, Hampton, C. Self-serving responses arising from discrepancies between explicit and implicit self-esteem. Self Identity. 2005;4(4), 311-330.

37. Faul, F, Erdfelder, E, Lang, AG, Buchner, A. G*Power 3: A flexible statistical power analysis program for the social, behavioral, and biomedical sciences. Behav Res Methods. 2007;39:175191.

38. Cohen, J. A power primer. Psychol Bull. 1992;112(1):155-159 Available from: https://doi.org/ 10.1037/0033-2909.112.1.155

39. Prezza, M, Trombaccia, FR, Armento, L. La scala dell'autostima di Rosenberg: Traduzione e validazione Italiana. Firenze: Giunti Organizzazioni Speciali. 1997

40. Braslow, MD, Guerrettaz, J, Arkin, RM, Oleson, KC. Self-doubt. Soc Personal Psychol Compass. 2012;6:470-482. Available from: https://doi.org/10.1111/j.1751-9004.2012.00441.x

41. Duru, E, Balkis, M. The roles of academic procrastination tendency on the relationships among self doubt, self esteem and academic achievement. Education and Science. 2014;39(173):274287

42. Richetin, J, Conner, M, Perugini, M. Not doing is not the opposite of doing: Implications for attitudinal models of behavioral prediction. Pers Soc Psychol Bull. 2011;37(1):40-54. 
43. Campbell, JD, Trapnell, PD, Heine, SJ, Katz, IM, Lavallee, LF, Lehman, DR. Self-concept clarity: Measurement, personality correlates, and cultural boundaries. J Pers Soc Psychol. 1996;70(1):141-156. Available from: https://doi.org/10.1037/0022-3514.70.1.141

44. Usborne, E, Taylor, DM. The role of cultural identity clarity for self-concept clarity, selfesteem, and subjective well-being. Pers Soc Psychol Bull. 2010;36(7):883-897.

45. $\mathrm{Wu}, \mathrm{CH}$. The relationship between attachment style and self-concept clarity: The mediation effect of self-esteem. Pers Individ Dif. 2009;47(1):42-46.

46. Maroiu, C, Maricuţoiu, LP, Sava, FA. Explicit self-esteem and contingencies of self-worth: The moderating role of implicit self-esteem. Pers Individ Dif. 2016;99:235-241. Available from: https://doi.org/10.1016/j.paid.2016.05.022

47. Moza, D, Maricuțoiu, L, Gavreliuc, A. Cross-lagged relationships between self-esteem, selfconstrual, and happiness in a three-wave longitudinal study. J Individ Differ. 2019;40(3).177185. Available from: https://doi.org/10.1027/1614-0001/a000290

48. The jamovi project. Jamovi (Version 1.2) [Computer Software] Retrieved from https://www.jamovi.org 2020, March 9, 2020.

49. Spencer, SJ, Jordan, CH, Logel, CE, Zanna, MP. Nagging doubts and a glimmer of hope: The role of implicit self-esteem in self-image maintenance. In: Tesser A, Wood JV, Stapel DA, editors. On building, defending and regulating the self: A psychological perspective. New York, NY: Psychology Press, Taylor \& Francis Group. 2005; p. 153-170. ISBN 1-84169-405-3 